\title{
Lidil
}

Revue de linguistique et de didactique des langues

$63 \mid 2021$

Littératie numérique et didactique des langues et des cultures

\section{Revue Enjeux, no 91}

CEDOCEF - Presses universitaires de Namur, hiver 2017, 172 p.

\section{Yves Reuter}

\section{CpenEdition}

\section{Journals}

Édition électronique

URL : http://journals.openedition.org/lidil/9053

DOI : 10.4000/lidil.9053

ISSN : 1960-6052

Éditeur

UGA Éditions/Université Grenoble Alpes

Édition imprimée

ISBN : 978-2-37747-283-3

ISSN : $1146-6480$

Référence électronique

Yves Reuter, «Revue Enjeux, no 91 », Lidil [En ligne], 63 | 2021, mis en ligne le 30 avril 2021, consulté le 30 avril 2021. URL : http://journals.openedition.org/lidil/9053 ; DOI : https://doi.org/10.4000/lidil.9053

Ce document a été généré automatiquement le 30 avril 2021.

(C) Lidil 


\title{
Revue Enjeux, n ${ }^{\circ} 91$
}

CEDOCEF - Presses universitaires de Namur, hiver 2017, 172 p.

\author{
Yves Reuter
}

\section{RÉFÉRENCE}

Revue Enjeux, nº 91, CEDOCEF - Presses universitaires de Namur, hiver 2017, 172 p.

1 Ce numéro de la revue Enjeux, « revue de formation continuée et de didactique du français » est le dernier numéro en format papier. Il comprend six articles sans relation thématique particulière.

2 Trois d'entre eux portent principalement, mais avec des éclairages différents, sur l'enseignement de la littérature. Jean-Louis Dumortier travaille ainsi sur l'effet idéologique du texte de fiction. Après un retour sur des textes essentiels sur cette question (Foucault, Sartre, Goldmann, Macherey, Hamon, Picard, Jouve...) et une critique particulièrement judicieuse des approches pédagogiques qui se font à l'aune d'un homme censé être universel et intemporel, Dumortier ouvre plusieurs pistes pédagogiques agrémentées d'exemples précis. On peut peut-être regretter ici que les références aux études sur le personnage, aux débats sur le roman policier (notamment à énigme) ou encore à des pratiques telles que le débat interprétatif ne soient pas plus présentes.

3 Nathalie Gillain et David Vrydaghs, de leur côté, proposent un modèle didactique (au sens des didacticiens du français de Genève) pour l'enseignement-apprentissage de la poésie intégrant la question de l'oralité, cela pour le troisième degré de l'enseignement du français en Fédération Wallonie - Bruxelles. Il s'agit ici de rompre avec les pratiques pédagogiques de lecture et/ou d'écriture essentiellement centrées sur l'écrit. Ce modèle d'ingénierie pédagogique, appuyé sur des analyses de la littérature théorique, des pratiques sociales de référence, des pratiques langagières des élèves (mais sans référence aux travaux de Marie-Claude Penloup) et des pratiques scolaires est, ici aussi, accompagné de quelques exemples d'activités possibles. 
4 Djamel Kadik, quant à lui, se réclamant des travaux de Peytard et d'Adam, propose un travail sur l'altération des textes littéraires. Il s'agit d'inviter les étudiants en classe de langue à reformuler, transcoder, transposer un texte d'un genre littéraire en un autre afin de les familiariser avec l'écriture littéraire et la littérature. Les dispositifs mis en place (situations, moyens et effets) sont étudiés de manière précise, même si, comme l'indique lui-même l'auteur, la question de l'évaluation demeure en suspens.

Deux autres articles portent sur des pans différents de l'enseignement du français. Jean- Louis Dumortier, infatigable didacticien au prisme particulièrement large, propose ainsi un second article, très stimulant sur l'enseignement de la forme passive afin de mieux l'adapter à l'objectif de développement des compétences de communication verbale. Après avoir développé une critique étayée des manières classiques de l'enseigner en notant, par exemple, que l'accent porté sur la conception d'une phrase passive dérivée conduit à faire opérer des transformations aux élèves sans véritablement se questionner sur les raisons de l'apparition de cette forme ou encore que les usages natifs ne procèdent pas par transformation, il déconstruit nombre de catégories en usage : opposition et dérivation; agent/patient; origine/aboutissement. Sa conclusion est qu'il est inutile et perturbant d'enseigner la voix passive à de jeunes élèves francophones, car ceux-ci ne sont embarrassés, ni en production, ni en réception. On remarquera qu'il ouvre pourtant lui-même des pistes intéressantes quant aux choix d'enseignement possibles. Mais il le fait en insistant sur la question du sens.

6 Benoit Sans rend compte d'un projet de formation, mené en partenariat avec l'Athénée Marguerite Yourcenar à Bruxelles en $3^{\mathrm{e}}$ secondaire, visant à réintroduire dans les classes des exercices de rhétorique inspirés de ceux qui avaient été inventés dans l'Antiquité pour former les citoyens (notamment changer de point de vue ou défendre deux points de vue opposés). Certains effets intéressants ont été constatés : intérêt des élèves, progrès dans les argumentations (plus riches et pertinentes, avec des stratégies plus conscientes), transfert dans d'autres situations (par exemple, les conseils de classe).

7 Un article, celui de Vincent Capt, Mathieu Depeursinghe et Sonya Florey, travaille sur un tout autre objet : les relations entre numérique et didactique du français. Quels sont les effets sur l'enseignement du français puisque les nouvelles technologies modifient temporalité et spatialité et mettent en question nombre de catégories telles espace scolaire/espace sociétal, écrit/oral, verbal/iconique, privé/public ? En retour, que peut la didactique du français? Il est en tout cas certain que cet article excède largement l'enseignement $d u$ français pour s'interroger sur fonctionnements et fonctions $d u$ numérique dans le contexte ainsi créé.

$8 \mathrm{Au}$ total, un numéro riche qui répond aux objectifs de la revue : articuler recherche théorique et réponses concrètes aux besoins des enseignants, même si, comme pour toute revue de didactique à l'heure actuelle, on peut se demander si tous les articles demeurent accessibles aux enseignants. 
AUTEUR

YVES REUTER

CIREL (ULR 4354), Université de Lille 